\title{
As Relações entre a Satisfação Conjugal e as Habilidades Sociais percebidas no Cônjuge
}

\author{
Aline Sardinha ${ }^{1}$ \\ Universidade Federal do Rio de Janeiro \\ Eliane Mary de Oliveira Falcone \\ Universidade Estadual do Rio de Janeiro \\ Maria Cristina Ferreira \\ Universidade Salgado de Oliveira
}

\begin{abstract}
RESUMO - As relações entre habilidades sociais percebidas pelo cônjuge e satisfação conjugal foram investigadas. Cinquenta casais $(\mathrm{n}=100)$, com idades entre 29 a 69 anos e tempo de união entre sete e 38 anos, responderam à Escala de Satisfação Conjugal, ao Inventário de Habilidades Sociais Conjugais e ao Questionário de Empatia Conjugal. A análise de regressão múltipla apontou forte relação entre a empatia do cônjuge e a satisfação conjugal, seguida pela expressão de sentimentos e defesa dos próprios direitos. O número de filhos se correlacionou inversamente com a satisfação e as mulheres foram percebidas como mais empáticas. As habilidades sociais, especialmente a empatia, parecem ser facilitadores da satisfação conjugal. Recomenda-se o desenvolvimento dessas habilidades no tratamento de casais em crise.
\end{abstract}

Palavras-chave: satisfação conjugal; casamento; habilidades sociais; empatia.

\section{The Relations between Marital Satisfaction and Social Skills Perceived in the Spouse}

\begin{abstract}
This study investigated the association between the social skills perceived by the spouse and marital satisfaction. Fifty couples ( $n=100)$, aged from 29 to 69 and with relationship lasting from seven to 38 years, were asked to fill in the Marital Satisfaction Scale, the Marital Social Skills Inventory and the Marital Empathy Questionnaire. Multiple regression analysis revealed that spouse's empathy was strongly correlated with marital satisfaction, followed by the expression of feelings and defense of own rights. The number of offspring was inversely correlated with satisfaction, and women were perceived by their partners as more empathic. The presence of social skills, particularly empathy, seems to be facilitators of marital satisfaction. Interventions directed to the development of social skills may be useful in the treatment of distressed couples.
\end{abstract}

Keywords: marital satisfaction; marriage; social skills; empathy.

O aumento paulatino do número de divórcios, tanto no Brasil quanto no mundo, revela dados preocupantes, com um índice de $60 \%$ nos EUA, de $40 \%$ na Inglaterra e de $25 \%$ no Brasil (Vila, 2002). Problemas de relacionamento entre casais e de insatisfação no casamento têm sido apontados como um dos maiores estressores da vida, levando a transtornos psiquiátricos e enfermidades físicas (Epstein \& Schlesinger, 1995; Fincham \& Horneffer, 1996; Granvold, 2004). O estresse no relacionamento conjugal pode levar à violência entre os parceiros, cujas consequências mais extremas são o homicídio ou o suicídio (Hamberger \& Holtzworth-Munroe, 2004). Além disso, perturbações conjugais também trazem consequências negativas para os filhos e familiares (Gottman \& Silver, 2000).

Os custos potenciais da crise conjugal e do divórcio têm feito com que esse tema ocupe um papel central na literatura psicológica sobre casais, motivando a busca de intervenções

1 Endereço para correspondência: Rua Visconde de Pirajá, 595/1205. Ipanema. Rio de Janeiro, RJ. CEP 22410-003. E-mail: alinesardinhapsi@gmail.com. para tratar o conflito entre os cônjuges (Fincham \& Horneffer, 1996). No campo da pesquisa, procura-se entender que fatores podem contribuir para um casamento feliz, uma vez que a satisfação conjugal fortalece o sistema imunológico do indivíduo, aumentando o seu tempo de vida (Gottman \& Silver, 2000). Todas essas constatações explicam por que a satisfação conjugal tem sido amplamente estudada (Oliveira, 2005), sendo esta compreendida como a satisfação do indivíduo com o relacionamento no geral e em seus aspectos específicos (Dela Coleta, 1989).

Em uma revisão de estudos, Norgren, Souza, Kaslow, Hammerschmidt e Sharlin (2004) encontraram que a satisfação com o casamento se relaciona a sentimentos de bem-estar, contentamento, companheirismo, afeição e segurança. Tais fatores promovem intimidade no relacionamento e decorrem da congruência entre as expectativas e aspirações dos cônjuges e a realidade vivenciada. Outros autores, como Arias e House (1998) e Dela Coleta (1992), reconhecem que a satisfação conjugal é um fenômeno complexo, além de sofrer a influência de diversas variáveis, tais como: características de personalidade, valores, atitudes e necessidades, sexo, 
momento do ciclo da vida familiar, presença de filhos, nível de escolaridade, nível socioeconômico, nível cultural, trabalho remunerado e experiência sexual anterior ao casamento. Entretanto, a capacidade para se comunicar parece constituir a base dessas variáveis.

Parker (2002, citado em Falcone \& Ramos, 2005) encontrou que os casamentos satisfeitos de longa data dependem de algumas habilidades de interação tais como: desenvolver capacidade para lidar com conflitos; consultar o parceiro na tomada de decisões; manter uma boa comunicação; cultivar valores tais como confiança, respeito, compreensão e equidade; desenvolver a intimidade sexual e psicológica. Em estudo realizado por Leichty, Willinhganz e Hart (2002, citado em Falcone \& Ramos, 2005), verificou-se que indivíduos que relataram maiores níveis de satisfação conjugal eram mais habilidosos socialmente e comunicavam-se mais adequadamente. Tais resultados são confirmados por diversos estudos que relacionam a capacidade de escuta e de resolução de conflitos ao aumento da satisfação conjugal e à redução do índice de divórcio (Bissonnette, Rusbult \& Kilpatrick, 1997; Caballo, 1987; Collins \& Collins, 1992; Davis \& Oathout, 1987; Goleman, 1995; Ickes, 1997; Karney \& Bradbury, 1995; Long \& Andrews, 1990; Villa, 2002). Terapeutas de base cognitivo-comportamental propõem que o desenvolvimento de habilidades sociais entre os casais em crise contribui significativamente para a maior eficácia da comunicação e da resolução de problemas conjugais (Beck, 1988/1995; Cordova \& Jacobson, 1999; Dattilio, 2004; Dattilio \& Padesky, 1995; Lima, 2003; Moraes \& Rodrigues, 2001).

A literatura sobre habilidades sociais vem se desenvolvendo a partir de constatações de sua influência na qualidade de vida e na saúde (Carneiro, Falcone, Clark, Del Prette \& Del Prette, 2007). Entretanto, não existe um consenso sobre a sua definição. Para Caballo (1991), o comportamento socialmente habilidoso corresponde ao:

Conjunto de comportamentos emitidos por um indivíduo em um contexto interpessoal que expressa sentimentos, atitudes, desejos, opiniões ou direitos deste indivíduo, de um modo adequado à situação, respeitando estes comportamentos nos outros e que geralmente resolve os problemas imediatos da situação, minimizando a probabilidade de problemas futuros. (p. 407)

A definição de Caballo refere-se ao comportamento assertivo, o qual é considerado pelo autor como sinônimo de habilidades sociais (Caballo, 1993). O termo assertividade envolve "defender os direitos pessoais e expressar pensamentos, sentimentos e crenças de forma honesta, direta e apropriada, sem violar os direitos da outra pessoa" (Lange \& Jakubowski, 1976, p. 7). Entretanto, alguns autores (e.g., Falcone, 1995, 2000; Del Prette \& Del Prette, 1999; Matos, 1997) afirmam que as habilidades sociais compreendem um repertório mais amplo de respostas, no qual se inclui o comportamento assertivo.

As habilidades sociais são consideradas de forma mais abrangente por Del Prette e Del Prette (2001), ao conceituálas como diferentes classes de comportamentos sociais que podem ser utilizados pelo indivíduo para lidar de maneira adequada com as demandas das situações interpessoais. Para os autores, o conjunto das habilidades necessárias para o bom relacionamento social inclui diferentes classes e subclasses, tais como habilidades de comunicação, assertivas, empáticas, de civilidade, de expressividade emocional, de trabalho etc. (Del Prette \& Del Prette, 2001).

Para Falcone e Ramos (2005), o conceito de comportamento socialmente competente deve incluir a capacidade de obter satisfação pessoal, por meio da expressão de sentimentos, da defesa dos próprios direitos (assertividade) e, ao mesmo tempo, da motivação genuína para compreender e atender às necessidades da outra pessoa (empatia). Além disso, componentes cognitivos, tais como a atenção, percepção, memória e processamento da informação estão também envolvidos no repertório apropriado de desempenho social. A partir desses componentes, o indivíduo pode saber quando e como se comportar adequadamente (Falcone, 2001).

A empatia "caracteriza-se pela habilidade em compreender, de forma acurada, os sentimentos e a perspectiva da outra pessoa, bem como de transmitir entendimento de tal maneira que esta se sinta verdadeiramente compreendida e acolhida" (Falcone, 1999, p. 24). O comportamento empático inclui: (a) um componente cognitivo, caracterizado por uma capacidade de compreender acuradamente a perspectiva e os sentimentos dos outros, referido como tomada de perspectiva; (b) um componente afetivo, caracterizado por sentimentos de compaixão/preocupação com a outra pessoa; e (c) um componente comportamental, entendido como manifestações verbais e não verbais de compreensão dos estados internos da outra pessoa (Falcone, 2000).

No contexto das interações sociais, a empatia é apreciada pelo interlocutor, que se sente valorizado, aceito, reconhecido e entendido (Nichols, 1995). Além disso, a empatia promove maior entendimento na interação (Barrett-Lennard, 1988; 1993; Burleson, 1985) e, em situações de conflito, reduz os atritos e a probabilidade de rompimento. A empatia possui, ainda, o poder de reduzir a raiva da outra pessoa, tornando-a mais disponível para ouvir, facilitando o diálogo de entendimento (Goleman, 1995; Nichols, 1995).

Entretanto, comportar-se de forma empática na maior parte do tempo, abrindo mão das próprias necessidades, pode levar à insatisfação e frustração. Assim, parece que a promoção de interações agradáveis e de satisfação pessoal consiste na integração das habilidades empáticas e assertivas (Falcone \& Ramos, 2005).

Os vários estudos empíricos e revisões teóricas atestando a importância das habilidades sociais na satisfação conjugal levam às seguintes questões: (a) o indivíduo que percebe o seu cônjuge como capaz de ouvir e compreender (empatia), assim como de expressar sentimentos e desejos adequadamente (assertividade), experimenta mais satisfações no casamento do que aquele que não tem essa percepção?; (b) as habilidades empáticas do cônjuge promovem mais satisfação em seu parceiro do que as habilidades assertivas? Tais questões motivaram a realização deste estudo que teve como objetivo avaliar o quanto a percepção das habilidades sociais do cônjuge pode influenciar a satisfação de um indivíduo com seu relacionamento conjugal. 
Tabela 1. Composição da amostra $(n=100)$.

\begin{tabular}{|c|c|c|c|c|}
\hline & Variáveis & Frequência (\%) & & \\
\hline \multirow[t]{2}{*}{ Sexo } & Masculino & 50,0 & & \\
\hline & Feminino & 50,0 & & \\
\hline \multirow[t]{3}{*}{ Escolaridade } & Ensino Médio & 25,0 & & \\
\hline & Superior Incompleto & 11,0 & & \\
\hline & Superior Completo & 60,0 & & \\
\hline \multirow[t]{4}{*}{ Número de casamentos } & Um & 88,0 & & \\
\hline & Dois & 8,0 & & \\
\hline & Três & 2,0 & & \\
\hline & Média & Desvio-Padrão & Mínimo & Máximo \\
\hline Idade & 48,5 anos & 9,6 & 29,0 & 69,0 \\
\hline Tempo de casamento & 22 anos & 8,7 & 7,0 & 38,0 \\
\hline Número de filhos & 2 & 1,0 & 0,0 & 6,0 \\
\hline
\end{tabular}

\section{Método}

\section{Participantes}

Foram avaliados os níveis de habilidades sociais e de satisfação conjugal de 100 participantes (50 casais). Definiu-se que os participantes deveriam estar casados há, pelo menos, sete anos e ter escolaridade mínima de Ensino Médio completo. As características sócio-demográficas da composição da amostra estão listadas na Tabela 1.

\section{Instrumentos}

A avaliação da satisfação conjugal foi realizada a partir da Escala de Satisfação Conjugal (Dela Coleta, 1989), em que o participante avalia sua própria satisfação com o casamento. Os inventários que medem habilidades sociais - Questionário de Empatia Conjugal (Oliveira, 2005) e Inventário de Habilidades Sociais Conjugais (Villa, 2002) - foram aplicados de forma a que as perguntas se relacionassem ao comportamento do cônjuge. Para isso, o Inventário de Habilidades Sociais Conjugais precisou ser gramaticalmente adaptado pelos pesquisadores de modo a que as respostas refletissem as habilidades do cônjuge, mantendo-se a estrutura e o conteúdo dos itens. Para o Questionário de Empatia Conjugal e para a Escala de Satisfação Conjugal existem duas versões, masculina e feminina, nas quais o gênero dos itens se altera, permanecendo, contudo, o mesmo conteúdo. Tal divisão em duas versões foi mantida neste estudo.

Escala de Satisfação Conjugal - ESC (Dela Coleta, 1989). Trata-se de uma escala de auto-informe, desenvolvida originalmente em língua espanhola, traduzida e validada para o português por Dela Coleta, em 1989. A escala contém 24 itens, cada um com três opções de resposta. Quanto maior a pontuação, maior a satisfação do participante com a relação conjugal. A escala é composta por três subescalas que medem os seguintes aspectos da relação conjugal: interação conjugal, aspectos emocionais e aspectos estruturais. Interação conjugal se refere à maneira como um cônjuge se comporta na interação com seu par, englobando aspectos da comunicação do casal e da frequência de comportamentos positivos e negativos na interação. Os itens que medem os aspectos emocionais dizem respeito à maneira como o companheiro expressa sentimentos e a como se comporta quando experimenta sentimentos negativos, como raiva e preocupação. O fator aspectos estruturais, por sua vez, é composto de itens que se relacionam com a maneira pela qual o parceiro organiza a própria vida e o impacto que tal organização tem no relacionamento conjugal. A versão traduzida teve sua estrutura fatorial validada por meio da análise fatorial. A escala alcançou um Alpha bastante alto tanto para a escala total $(\alpha=0,91)$, quanto para suas subescalas: interação conjugal $(\alpha=0,86)$, aspectos emocionais $(\alpha=0,81)$ e aspectos estruturais $(\alpha=0,79)$.

Questionário de Empatia Conjugal - QEC (Oliveira, 2005). Trata-se de um questionário auto-aplicável, composto por 36 itens, contendo elementos cognitivos, afetivos e comportamentais observados nas pesquisas de relações conjugais, que busca avaliar como o respondente percebe o comportamento do companheiro em relação a si próprio. Cada item tem uma pontuação que varia entre 1 (nunca) e 4 (sempre). Quanto maior a pontuação, maior a empatia conjugal do parceiro. Este questionário teve sua consistência interna verificada e sua estrutura unifatorial validada pelo método da análise fatorial, obtendo $\alpha=0,95$ para a escala total.

Inventário de Habilidades Sociais Conjugais - IHSC (Villa, 2002). É baseado no IHS-Del Prette (Del Prette \& Del Prette, 2001). O IHSC é semelhante ao IHS-Del Prette, com algumas formulações para torná-lo mais específico às 
situações próprias do relacionamento conjugal, resultando em um instrumento com 32 itens. A análise da estrutura fatorial do IHSC foi feita pelo método Alpha, com rotação Varimax, resultando em cinco fatores (comunicação assertiva, expressão de sentimento positivo/agrado, expressão de desacordo/desagrado, desinibição/espontaneidade e controle da agressividade), $\mathrm{O}$ coeficiente Alpha, que determina a consistência interna dos fatores variou de 0,85 a 0,42 . Por meio do Alpha de Cronbach total $(0,81)$, constatou-se que o IHSC possui uma boa consistência interna, no que concerne à escala total.

\section{Procedimento}

A pesquisa obteve aprovação no Comitê de Ética em Pesquisa da Universidade do Estado do Rio de Janeiro (Parecer 028/2006). O estudo foi realizado em uma clínica médica, Clínica de Medicina do Exercício, situada em Copacabana, Rio de Janeiro. Foram entregues, a cada participante, um Termo de Consentimento Livre e Esclarecido, os três instrumentos de pesquisa e uma folha contendo perguntas para a caracterização da amostra (idade, sexo, tempo de casamento, número de filhos, número de casamentos e grau de instrução). Não houve tempo-limite para a resposta aos questionários e foi permitido que os participantes levassem os inventários para casa, devolvendo após alguns dias. Os casais foram instruídos a responder às questões individualmente e não houve nenhum tipo de devolução dos resultados aos participantes.

\section{Análise de dados}

Foram realizadas análises estatísticas descritivas com o objetivo de caracterizar o grupo estudado, bem como análises estatísticas sobre as relações entre as variáveis pesquisadas. O software utilizado foi o SPSS (versão 11.0).

Optou-se por reavaliar a estrutura fatorial de dois instrumentos utilizados, o IHSC e o QEC, a partir dos dados obtidos nesta amostra, a fim de verificar a estrutura unifatorial do QEC e de obter, no IHSC, uma estrutura fatorial que apresentasse maior validade em relação à amostra deste estudo.
O procedimento utilizado foi o de análise fatorial por meio do método dos componentes principais.

Foram inicialmente calculadas as médias obtidas pelos participantes em cada fator, de maneira que, quanto maior o resultado, maior o grau da característica mensurada pelo fator. Em seguida, foram calculados as médias, os desviospadrão e os coeficientes de correlação de Pearson de cada par de fatores.

Posteriormente, foram realizadas análises de regressão linear múltipla, adotando-se as variáveis sócio-demográficas (idade, sexo, escolaridade, número de casamentos, número de filhos e tempo de casamento) e as dimensões do QEC e do IHSC como preditoras (variáveis independentes) e as três modalidades de satisfação conjugal (interação conjugal, aspectos emocionais e aspectos estruturais) como variáveis critério (dependentes).

No método de regressão linear múltipla, cada bloco de variáveis independentes é introduzido na equação em uma ordem predeterminada pelo pesquisador. Nesse sentido, para o controle de seus efeitos na satisfação conjugal, as variáveis sócio-demográficas foram incluídas no primeiro bloco, enquanto no segundo bloco foram adicionadas as dimensões do IHSC e do QEC, verificando-se a contribuição da adição de cada bloco e variáveis no modelo pelo incremento do $\mathrm{R}^{2}$. Tais procedimentos foram realizados separadamente para cada uma das variáveis critério.

\section{Resultados}

Na análise fatorial do QEC, de acordo com os dados da amostra, verificou-se que a estrutura mais apropriada seria uma estrutura unifatorial, englobando itens que medem características cognitivo-comportamentais da empatia e descartando outros que não se encaixavam estatisticamente em nenhum fator, ficando o questionário com 24 itens, todos com cargas fatoriais acima de 0,45. Assim, o Alpha obtido para esta estrutura foi de 0,94 .

A nova estrutura fatorial encontrada para o IHSC consistiu em três fatores, denominados neste estudo: (1) Tolerância e escuta empática; (2) Expressão de sentimentos; e (3) Defesa dos próprios direitos. Os coeficiente Alpha dos fatores obtidos foram, respectivamente: $0,81,0,71$ e 0,90 . Alguns itens foram

Tabela 2. Médias e desvios-padrão das pontuações obtidas nos fatores das escalas.

\begin{tabular}{llcc}
\hline Escala & Fatores & Média & Desvio Padrão \\
\hline Escala de Satisfação Conjugal (ESC) & Interação conjugal & 2,53 & 0,49 \\
& Aspectos emocionais & 2,17 & 2,44 \\
& Aspectos estruturais & 2,95 & 0,46 \\
\hline Questionário de Empatia Conjugal (QEC) & & 3,43 & 0,57 \\
\hline Inventário de Habilidades Sociais Conjugais (IHSC) & Tolerância e escuta empática & 3,93 \\
& Expressão de sentimentos & 0,86 \\
& Defesa dos próprios direitos & 3,62 \\
\hline
\end{tabular}


Tabela 3. Diferenças quanto ao sexo nas diferentes sub-escalas

\begin{tabular}{|c|c|c|c|c|c|}
\hline Escala & Sexo & Média & $\begin{array}{l}\text { Desvio } \\
\text { Padrão }\end{array}$ & $t$ & $p$ \\
\hline \multirow[t]{2}{*}{ Interação conjugal } & $\mathrm{F}$ & 2,44 & 0,51 & $-1,81$ & 0,07 \\
\hline & M & 2,62 & 0,45 & $-1,81$ & 0,07 \\
\hline \multirow[t]{2}{*}{$\begin{array}{l}\text { Aspectos } \\
\text { emocionais }\end{array}$} & $\mathrm{F}$ & 2,11 & 0,63 & $-1,01$ & 0,32 \\
\hline & M & 2,22 & 0,52 & $-1,01$ & 0,32 \\
\hline \multirow[t]{2}{*}{$\begin{array}{l}\text { Aspectos } \\
\text { estruturais }\end{array}$} & $\mathrm{F}$ & 2,38 & 0,48 & $-1,45$ & 0,15 \\
\hline & M & 2,51 & 0,43 & $-1,45$ & 0,15 \\
\hline \multirow[t]{2}{*}{ QEC } & $\mathrm{F}$ & 2,81 & 0,61 & $-2,64$ & $0,01^{*}$ \\
\hline & M & 3,10 & 0,49 & $-2,64$ & $0,01^{*}$ \\
\hline \multirow[t]{2}{*}{$\begin{array}{l}\text { Tolerância e } \\
\text { escuta empática }\end{array}$} & $\mathrm{F}$ & 3,45 & 0,94 & 0,20 & 0,84 \\
\hline & M & 3,42 & 0,79 & 0,20 & 0,84 \\
\hline \multirow[t]{2}{*}{$\begin{array}{l}\text { Expressão de } \\
\text { sentimentos }\end{array}$} & $\mathrm{F}$ & 3,89 & 0,67 & $-0,82$ & 0,14 \\
\hline & M & 3,98 & 0,49 & $-0,82$ & 0,41 \\
\hline \multirow[t]{2}{*}{$\begin{array}{l}\text { Defesa dos } \\
\text { próprios direitos }\end{array}$} & $\mathrm{F}$ & 3,60 & 0,87 & $-0,27$ & 0,79 \\
\hline & M & 3,65 & 0,82 & $-0,27$ & 0,79 \\
\hline
\end{tabular}

excluídos por não se encaixarem estatisticamente na estrutura fatorial, ficando o instrumento com 25 itens.

A estrutura fatorial original da ESC foi mantida. Entretanto, foram calculados os coeficientes Alpha dos fatores para a presente amostra. $\mathrm{O}$ fator 1 , interação conjugal, obteve um Alpha igual a 0,90; o fator 2, aspectos emocionais, atingiu um Alpha de 0,87; e o fator 3, aspectos estruturais, alcançou um Alpha igual a 0,84 .

Foram calculados ainda as médias e os desvios-padrão das respostas dadas pelos participantes aos itens de cada uma das escalas. Nos fatores da ESC, as respostas poderiam variar entre 1 e 3; no QEC, entre 1 e 4; e, nas escalas do IHSC, entre 1 e 5 . Na Tabela 2 estão as médias e desvios-padrão para cada escala.
Além disso, as médias das respostas dadas pelos participantes a cada uma das escalas foram comparadas quanto ao sexo. Nessa análise, foi encontrada uma diferença significativa entre os sexos apenas nas respostas ao QEC, com os homens tendendo a avaliar suas parceiras como mais empáticas do que estas os avaliaram (Tabela 3).

Posteriormente, foram calculadas também as correlações de Pearson entre os fatores das medidas de habilidades sociais e os fatores da ESC, como mostra a Tabela 4. A partir desse cálculo, observa-se que o QEC apresentou as maiores correlações com as escalas que mediam satisfação conjugal.

A seguir, foi realizada a análise de regressão múltipla para cada um dos fatores da ESC. A análise foi feita em dois blocos, sendo o primeiro bloco constituído das variáveis sócio-demográficas da amostra (sexo, idade, número de filhos, número de casamentos e escolaridade) e o segundo bloco, dessas variáveis acrescidas das subescalas que mediam habilidades sociais (QEC, Tolerância e escuta empática, Expressão de sentimentos e Defesa dos próprios direitos). Abaixo, encontram-se os resultados para cada fator da satisfação conjugal.

a) Interação conjugal. As variáveis sócio-demográficas (primeiro bloco) explicaram $13 \%$ da variância $\left(\mathrm{F}_{(5,91)}=2,81\right.$; $p<0,021)$ e apenas o número de filhos afetou significativa e negativamente a satisfação conjugal $(\beta=-0,31 ; t=-2,73$; $p<0,008)$. A entrada das medidas de habilidades sociais (segundo bloco) na equação se mostrou capaz de explicar $62 \%$ da variância $\left(\mathrm{F}_{(4.87)}=55,51 ; p<0,000\right)$, indicando que as habilidades sociais influenciam significativamente a interação conjugal.

Nesse modelo, a medida que mais contribuiu para a explicação da interação conjugal foi a empatia (QEC), que se correlacionou positiva e significativamente com esse fator da satisfação conjugal $(\beta=0,64, t=6,52 ; p<0,00)$, seguida pelo número de filhos $(\beta=-0,18 ; t=-2,74 ; p<0,007)$, que se correlacionou negativa e significativamente com a interação conjugal, e pela expressão de sentimentos $(\beta=0,148 ; t=2,47$; $p<0,015)$, que também apresentou uma correlação positiva significativa com essa variável.

Tais dados indicam que quanto maior o nível de empatia percebida no cônjuge, melhor será a interação conjugal. Por outro lado, a qualidade da interação conjugal decai com o aumento do número de filhos. Finalmente, níveis mais elevados de expressão de sentimentos do cônjuge, percebidos pelo seu par, também se relacionam com mais qualidade da interação conjugal, porém, em menor grau do que a empatia conjugal.

Tabela 4. Correlação de Pearson entre as escalas.

\begin{tabular}{lccc}
\hline \multirow{2}{*}{ Habilidades sociais } & \multicolumn{3}{c}{ Satisfação conjugal } \\
\cline { 2 - 4 } & Interação conjugal & Aspectos emocionais & Aspectos estruturais \\
\hline Empatia (QEC) & 0,82 & 0,60 & 0,70 \\
Tolerância e escuta empática & 0,60 & 0,52 & 0,51 \\
Expressão de sentimentos & 0,44 & 0,40 & 0,29 \\
Defesa dos próprios direitos & 0,60 & 0,50 & 0,49 \\
\hline
\end{tabular}


b) Aspectos emocionais. As variáveis sócio-demográficas, que formavam o primeiro bloco, foram responsáveis pela explicação de $2 \%$ da variância nesse modelo $\left(\mathrm{F}_{(5,91)}=0,63\right.$; $p<0,674)$, enquanto a adição das medidas de habilidades sociais (segundo bloco) no cálculo explicou $69 \%$ dessa variância $\left(\mathrm{F}_{(4,87)}=18,34 ; p<0,000\right)$. Isso significa que somente as variáveis sócio-demográficas não podem explicar de maneira significativa a variação dos aspectos emocionais da satisfação conjugal e que as medidas de habilidades sociais, por outro lado, influenciam significativamente esse fator.

Nesse bloco, as variáveis que se correlacionaram positiva e significativamente com os aspectos emocionais foram a expressão de sentimentos $(\beta=0,25 ; t=2,84 ; p<0,006)$ e a defesa dos próprios direitos $(\beta=0,22 ; t=2,12 ; p<0,037)$. Em outras palavras, quanto mais um indivíduo percebe o seu cônjuge expressando sentimentos e agindo de modo a defender seus próprios direitos, mais satisfeito estará esse membro do casal com os aspectos emocionais do seu parceiro.

c) Aspectos estruturais. No que se refere aos aspectos estruturais do parceiro que contribuem para a satisfação conjugal, a análise de regressão múltipla apresentou um grau de $7 \%$ da variância explicada somente pelas variáveis sócio-demográficas (primeiro bloco) $\left(\mathrm{F}_{(5,91)}=1,39 ; p<0,236\right)$, enquanto o segundo bloco (adicionando as medidas de habilidades sociais) foi responsável por $47 \%$ da variância encontrada $\left(\mathrm{F}_{(4.87)}=22,20 ; p<0,000\right)$. Nesse modelo, apenas a empatia (QEC) correlacionou-se significativa e positivamente com a variável critério $(\beta=0,65 ; t=4,83 ; p<0,000)$. Assim, somente o grau de empatia percebida no cônjuge influenciou diretamente a satisfação com os aspectos estruturais do parceiro.

\section{Discussão}

O presente estudo procurou responder a algumas questões a respeito do impacto das habilidades sociais percebidas no cônjuge na satisfação conjugal de seu parceiro. Verificou-se, a partir dos dados obtidos, que a empatia se relaciona direta e significativamente com todos os aspectos da satisfação conjugal. Assim, quanto maior o grau de empatia percebido no cônjuge, melhor a interação conjugal e maiores os níveis de satisfação alcançados com os aspectos emocionais e estruturais do cônjuge.

Tais dados confirmam os estudos que enunciam que a empatia contribui para maior qualidade no casamento, na medida em que promove maior entendimento na interação, diminuindo a possibilidade de rompimento, intensificando o afeto, aliviando a angústia da outra pessoa, bem como aumentando a auto-estima e a aceitação do cônjuge que manifesta empatia (Barrett-Lennard, 1988; 1993; Bissonnette \& cols., 1997; Burleson, 1985; Goleman, 1995; Long \& Andrews, 1990; Nichols, 1995; Oliveira, 2005).

Assim, sugere-se que a empatia influencia diretamente a interação conjugal, na medida em que, quando um parceiro procura ver os fatos sob a ótica do outro, muitos atritos na interação podem ser evitados e a solução de problemas de interação se torna mais fácil. Além disso, a satisfação com os aspectos estruturais do cônjuge mostra-se mais elevada, uma vez que o indivíduo, ao adotar a perspectiva do parceiro, consegue organizar sua vida de maneira a levar em consideração as necessidades de seu cônjuge. Ainda, a adoção de uma postura empática reduz a raiva, tornando o parceiro mais disponível para ouvir, facilitando o diálogo de entendimento (Goleman, 1995; Nichols, 1995), o que provavelmente levará a uma maneira mais adequada de lidar com sentimentos negativos, contribuindo para maior satisfação quanto aos aspectos emocionais do parceiro no relacionamento.

Os dados desta pesquisa sugerem também que um cônjuge capaz de expressar seus sentimentos contribui para maior satisfação de seu par com o relacionamento conjugal. Esse fator influencia a satisfação conjugal em menor grau do que a empatia, mas ainda assim, de maneira significativa. A literatura indica que, no cotidiano, a expressão assertiva dos desejos, sentimentos e necessidades facilita a solução de problemas interpessoais, aumenta o senso de auto-eficácia e a auto-estima, melhora a qualidade dos relacionamentos e promove a tranquilidade (Falcone, 1999). Ainda, a partir desses resultados, é possível hipotetizar que compreender como o parceiro se sente, por meio da expressão adequada de sentimentos por parte deste, facilita que o cônjuge emita comportamentos e tenha atitudes que contribuam para a satisfação conjugal.

De acordo com Taganelli (2003), as dificuldades em interpretar o comportamento e os sentimentos do outro levam ao fracasso do relacionamento. Assim, na medida em que um indivíduo se expressa satisfatoriamente, seu cônjuge pode atender suas necessidades de maneira mais acertada e esse cônjuge pode, ainda, sentir-se mais satisfeito quando recebe um feedback sincero sobre suas ações e pode ajustá-las de forma a agradar seu parceiro.

Parece razoável pensar que o sentimento de ser compreendido, acolhido e validado pelo cônjuge empático promove uma sensação de aceitação e valorização, levando à satisfação de um indivíduo com seu casamento. É possível que o próprio indivíduo que empatiza se sinta mais satisfeito ao promover a satisfação conjugal de seu parceiro, pelas razões acima descritas. Além disso, aquele que consegue expressar adequadamente seus sentimentos, provavelmente também se sentirá mais aliviado e feliz ao provocar bem estar em seu cônjuge. Nesse sentido, é possível concluir que tanto a empatia quanto a expressão de sentimentos do parceiro atuam diretamente na satisfação conjugal de um indivíduo.

O estudo também permite concluir que, em certos contextos, a combinação de expressões assertivas e empáticas gera efeito ainda maior sobre a satisfação com o casamento do que cada uma delas isoladamente. Tal hipótese confirma as afirmações de Falcone (2003), em que somente a expressão de sentimentos e necessidades não é suficiente para um relacionamento interpessoal satisfatório, sendo necessária também a empatia para maximizar a qualidade dos relacionamentos.

Os resultados do estudo mostraram que o número de filhos estava inversamente relacionado à qualidade da interação conjugal. Apesar de não ter sido avaliado o impacto dessas variáveis diretamente sobre o grau de habilidades sociais de um indivíduo, pode-se hipotetizar que o estresse causado pelo maior número de filhos demande mais esforço e habilidades dos cônjuges. Esse resultado é coerente com dados de Ellison e Hall (2003), que apontam que múltiplos filhos têm 
impacto negativo em diversos aspectos da qualidade de vida, incluindo a satisfação conjugal e o estresse emocional dos pais. Em outro estudo, Ellison e colaboradores encontraram que para cada filho adicional a partir do primeiro, as chances de piora na qualidade de vida dos pais e de toda a família mais do que dobram (Ellison \& cols., 2005).

Finalmente, não foram observadas diferenças significativas entre os gêneros em relação à satisfação conjugal. Contudo, houve diferença significativa entre os sexos em relação à empatia percebida no cônjuge. Os homens tenderam a avaliar suas parceiras como mais empáticas do que foram avaliados pelas mesmas. Assim, pode-se considerar que, nessa amostra, as mulheres atingiram maiores níveis de empatia do que os homens. Tal dado confirma o estudo de Graham e Ickes (1997, citado por Falcone, 1999), que afirmam terem as mulheres maiores níveis de empatia. Essa diferença pode se dever, de acordo com Falcone (1999), tanto a uma predisposição biológica moldada pela necessidade evolutiva de decodificar acuradamente os estados emocionais e necessidades dos bebês, quanto a fatores culturais, uma vez que, em nossa sociedade, as mulheres são treinadas a estarem atentas às necessidades dos outros. Por outro lado, no que se refere à expressão de sentimentos, não houve, nessa amostra, diferenças significativas entre os sexos.

Concluindo, o estudo confirma pesquisas anteriores que atestam ser a satisfação conjugal influenciada pelo nível elevado de habilidades sociais dos cônjuges. Espera-se que esses resultados possam contribuir para futuros estudos, visando à melhoria da satisfação conjugal e da qualidade de vida.

\section{Referências}

Arias, I., \& House, A. S. (1998). Tratamiento cognitivoconductal de los problemas de pareja. Em V. E. Caballo (Org.), Manual para el tratamiento cognitivo-conductal de los transtornos psicológicos, Vol.2 (pp. 553-577). Madrid: Siglo Veintiuno.

Barrett-Lennard, G. T. (1988). Listening. Person-Centered Review, 3 (4), 410-425.

Barrett-Lennard, G. T. (1993). The phases and focus of empathy. The British Journal of Medical Psychology, 66(1), 3-14.

Beck, A. T. (1995). Para além do amor. (P. Fróes, Trad.) Rio de Janeiro: Rosa dos Tempos (Trabalho original publicado em 1988).

Bissonnette, V. L., Rusbult, C. E., \& Kilpatrick, S. D. (1997). Empathic accuracy and marital conflict resolution. Em W. Ickes (Org.), Empathic accuracy (pp.251-281). New York: Gilford.

Burleson, B. R. (1985). The production of comforting messages: Social cognitive foundations. Journal for Language and Social Psychology, 4, 253-273.

Caballo, V. E. (1987). Teoría, evaluación y entrenamiento de las habilidades sociales. Valencia: Promolibro.

Caballo, V. E. (1991). El entrenamiento en habilidades sociales. Em V. E. Caballo (Org.), Manual de técnicas de terapia y modificación de conducta (pp. 403-443). Madrid: Siglo Veintiuno.

Caballo, V. E. (1993). Manual de evaluación y entrenamiento de las habilidades sociales. Madrid: Siglo Veintiuno.

Carneiro, R. S., Falcone, E., Clark, C., Del Prette, Z. A. P., \& Del Prette, A. (2007). Qualidade de vida, apoio social e depressão em idosos: relação com habilidades sociais. Psicologia: Reflexão e Crítica, 20, 29-237.
Collins, J., \& Collins, M. (1992). Social skills training and the professional helper. New York: Willey.

Cordova, J. V., \& Jacobson, N. S. (1999). Crise de casais. Em D. H. Barlow (Org.), Manual clínico dos transtornos psicológicos ( $2^{\mathrm{a} e d .)}$ (pp.535-567). Porto Alegre: Artmed.

Dattilio, F. M. (2004). Casais e famílias. Em P. Knapp (Org.), Terapia cognitivo-comportamental na prática psiquiátrica (pp. 377-401). Porto Alegre: Artmed.

Dattilio, F. M., \& Padesky, C. A. (1995). Terapia cognitiva com casais. Porto Alegre: Artmed.

Davis, M. H., \& Oathout, H.A. (1987). Maintenance of satisfaction in romantic relationships: Empathy and relational competence. Journal of Personality and Social Psychology, 53, 397-410.

Del Prette, Z. A. P., \& Del Prette, A. (1999). Psicologia das habilidades sociais - terapia e educação. Petrópolis: Vozes.

Del Prette, Z. A. P., \& Del Prette, A. (2001). Inventário de Habilidades Sociais (IHS-Del Prette): manual de aplicação, apuração e interpretação. São Paulo: Casa do Psicólogo.

Dela Coleta, M. F. (1989). A medida da satisfação conjugal: adaptação de uma escala. Psico, 18(2), 90-112.

Dela Coleta, M. F. (1992). Locus de controle e satisfação conjugal. Psicologia: Teoria e Pesquisa, 8, 243-252.

Ellison, M. A., \& Hall, J. E. (2003). Social stigma and compounded losses: Quality-of-life issues for multiple-birth families. Fertility and Sterility, 80, 405-414.

Ellison, M. A., Hotamisligil, S., Lee, H., Rich-Edwards, J. W., Pang, S. C., \& Hall J. E. (2005). Psychosocial risks associated with multiple births resulting from assisted reproduction. Fertility and Sterility, 83, 1422-1428.

Epstein, N. B., \& Schlesinger, S. E. (1995). Casais em crise Em F. M. Dattilio \& A. Freedman (Orgs.), Estratégias cognitivocomportamentais de intervenção em situações de crise ( $\left.2^{\mathrm{a}} \mathrm{ed}.\right)$ (pp.300-316). Porto Alegre: Artmed.

Falcone, E. M. O. (1995). Grupos. Em B. Rangé (Org.), Psicoterapia comportamental e cognitiva: pesquisa, prática, aplicações e problemas, Vol. 2 (pp. 159-169). Campinas: Editorial Psy.

Falcone, E. M. O. (1999) A avaliação de um programa de treinamento da empatia com universitários. Revista Brasileira de Terapia Comportamental e Cognitiva, 1, 23-32.

Falcone, E. M. O. (2000). Habilidades sociais: para além da assertividade. Em R. C. Wielenska (Org.), Sobre comportamento e cognição: questionando e ampliando a teoria e as intervenções clínicas em outros contextos (pp. 211-221). Santo André: SET.

Falcone, E. M. O. (2001). Uma proposta de um sistema de classificação das habilidades sociais. Em H. J. Guilhardi, M. B. B. P. Madi, P. P.Queiroz \& M. C. Scoz (Orgs.), Sobre comportamento e cognição: expondo a variabilidade (pp. 195-209). Santo André: ESETEC.

Falcone, E. M. O. (2003). Empatia. Em C. N. de Abreu \& M. Roso (Orgs.), Psicoterapias cognitiva e construtivista. Novas fronteiras da prática clínica (pp.275-287). Porto Alegre: Artmed.

Falcone, E. M. O., \& Ramos, D. M. (2005). A atribuição como componente cognitivo das habilidades sociais e seu impacto na satisfação conjugal. Em H. J. Guilhardi \& N. C. Aguirre (Orgs.), Sobre comportamento e cognição: questionando e ampliando a teoria e as intervenções clínicas em outros contextos (pp. 182-191). Santo André: SET.

Fincham, F. D., \& Horneffer, K. (1996). Conflictos de pareja. Em V. E. Caballo, G. Buela-Casal \& J. A. Carrobles (Orgs.), Manual de psicopatologia y trastornos psiquiátricos, Vol. 2 (pp. 551-587). Madrid: Siglo Veintiuno. 
Goleman, D. (1995). Inteligência emocional: a teoria revolucionária que redefine o que é ser inteligente. Rio de Janeiro: Objetiva.

Gottman, J., \& Silver, N. (2000). Sete principios para o casamento dar certo. Rio de Janeiro: Objetiva.

Granvold, D. K. (2004). Divórcio. Em F. M. Dattilio \& A. Freedman (Orgs.), Estratégias cognitivo-comportamentais de intervenção em situações de crise ( $2^{\mathrm{a}} \mathrm{ed}$.) (pp. 300-316). Porto Alegre: Artmed.

Hamberger, L. K., \& Holtzworth-Munroe, A. (2004). Parceiros violentos. Em F. M. Dattilio \& A. Freeman (Orgs.), Estratégias cognitivo-comportamentais de intervenção em situações de crise (2 ed.) (pp.281-299). Porto Alegre: Artmed.

Ikes, W. (1997). Introduction. Em W. Ikes (Org.), Empathic accuracy (pp. 282-310). New York: Guilford.

Karney, B. R., \& Bradbury, T. N. (1995). The longitudinal course of marital quality and stability: A review of theory, method, and research. Psychological Bulletin, 118, 3-34.

Lange, A., \& Jakubowski, P. (1976). Responsible assertive behavior. Illinois: Research Press.

Lima, M. V. O. (2003). Terapia de casal: enfoque cognitivo. Em C. N. Abreu \& M. Roso (Orgs.), Psicoterapias cognitiva e construtivista: novas fronteiras da prática clínica (pp. 229-236). Porto Alegre: Artmed.

Long, E. C. J., \& Andrews, D. W. (1990). Perspective taking as a predictor of marital adjustment. Journal of Personality and Social Psychology, 59, 126-131.

Matos, M. G. (1997). Comunicação e gestão de conflitos na escola. Lisboa: Edições FMH.
Moraes, C. G. A., \& Rodrigues, A. S. (2001). Terapia de casais. Em B. Rangé (Org.), Psicoterapias cognitivo-comportamentais: um diálogo com a psiquiatria (pp.513-522). Porto Alegre: Artmed.

Nichols, M. P. (1995). The lost art of listening. New York: Guilford.

Norgren, M. B. P., Souza, R. M., Kaslow, F., Hammerschmidt, H., \& Sharlin, S. A. (2004). Satisfação conjugal em casamentos de longa duração: uma construção possível. Estudos de Psicologia, 9, 575-584.

Oliveira, M. G. S. (2005). A avaliação da empatia e da satisfação conjugal: um estudo preliminar. Dissertação de Mestrado, Universidade do Estado do Rio de Janeiro, Rio de Janeiro.

Tanganelli, M. S. (2003). Você me estressa, eu estresso você. Em M. Lipp (Org.), O stress está dentro de você (pp.155-168). São Paulo: Contexto.

Villa, M. B. (2002). Habilidades sociais com conjugais em casais de diferentes filiações religiosas. Dissertação de Mestrado, Universidade de São Paulo, São Paulo.
Recebido em 28.02.07

Primeira decisão editorial em 18.05.09

Versão final em 08.06.09

Aceito em 15.06.09 